\title{
Correlación clínica e inmunohistopatológica de la nefropatía lúpica en un centro de referencia del Caribe colombiano durante los años 2012 a 2013
}

\author{
Alonso R Arroyo C $\mathrm{C}^{1}$, Raúl García ${ }^{2}$, Gustavo Aroca ${ }^{2}$, Andres Cadena ${ }^{2}$, Jorge Acosta ${ }^{3}$ \\ ${ }^{1}$ Universidad del Norte, ${ }^{2}$ Clínica de la Costa, ${ }^{3}$ Universidad del Norte. Barranquilla, Colombia
}

\begin{abstract}
Resumen
Objetivos: Determinar el comportamiento de la nefropatía lúpica (NL) en un grupo poblacional de un centro de referencia del Caribe colombiano, durante los años 2012 y 2013, analizando los hallazgos clínicos y de laboratorio más prevalentes y su relación con los hallazgos inmunohistopatológicos, definir la clase de NL con mayor prevalencia y el dato clínico o paraclínico más correlacionado con esta.

Métodos: Estudio descriptivo, retrospectivo, transversal, realizado desde el 1 de enero de 2012 al 31 de diciembre de 2013, que incluyó a 53 pacientes con lupus eritematoso sistémico (LES), según los criterios diagnósticos del Colegio Americano de Reumatología, con manifestaciones de compromiso renal.

Resultados: En nuestra población de estudio del Caribe colombiano, la NL predomina más en mujeres y la clase IV representa el $66,03 \%$ del total de casos analizados, en los cuales hubo más presentación de hematuria, hipertensión y proteinuria con patrón nefrótico, al igual que mayores niveles de creatinina sérica. A pesar de la falta de datos, ninguna de las variables clínicas o paraclínicas fue específica para cierta clase de NL y con relación a la inmunomarcación, las cadenas lambda se presentaron positivas en el $100 \%$ de los casos y tan solo el C4 y el fibrinógeno fueron específicos de la NL clase IV.

Conclusiones: La NL clase IV es la más prevalente y con mayor porcentaje de predominio de las variables como hematuria, proteinuria, hipertensión, niveles de creatinina y BUN séricos elevados y, a su vez, no son específicos para cierta clase de NL en nuestra población.
\end{abstract}

Palabras clave: Nefritis lúpica, Histopatología.

\section{Clinical and immune histopathological relationship of lupic nephropathy in a reference center of the Colombian caribbean between 2012 and 2013}

\begin{abstract}
Objectives: Determine the behavior of lupic nephropathy (LN) in a group of population of a reference center of the Colombian Caribbean between 2012 and 2013 by analyzing the most prevalent clinical and laboratory findings and their relationship with immune histopathological findings, define the type of lupic nephritis with the most prevalent and related clinical or paraclinical finding.
\end{abstract}


Methods: Descriptive, retrospective, transversal study, between January 1st 2012 and December 31st 2013 which included 53 patients with systemic lupus erythematousus that meet the American Rheumatology College criteria with renal involvement.

Results: In our target population of the Colombian Caribbean the LN is mostly common among women and the IV type represents $66.03 \%$ of the total analyzed cases, which didn't present hematuria, hypertension, proteinuria with a nefrotic pattern, and higher levels of seric creatinine. Despite the lack of data, none of the clinical or paraclinical variables was specific for a type of $\mathrm{LN}$, and in the immune staining, the lamda chains were positive in $100 \%$ of the cases and only the C4 and the fibrinogen were specific for the type IV LN.

Conclusions: The type IV LN is the most prevalent and has the highest percentage of predominance of the variables hematuria, proteinuria, hypertension, creatinine and BUN increased seric levels, which at the same time aren't specific for certain types of LN in our population.

Key Words: Lupic Nephropathy, Histopathology.

\section{Introducción}

E 1 compromiso renal en los pacientes con lupus eritematoso sistémico (LES) es muy alto con relación al compromiso de otros órganos. La literatura describe que hasta $50 \%$ de estos pacientes pueden cursar con nefritis lúpica (NL) de acuerdo con sus características demográficas, con una incidencia de hasta el $55 \%$ en asiáticos, $51 \%$ en africanos, $43 \%$ en hispanos y $14 \%$ en caucásicos ${ }^{1,2}$.

Estudios en población europea establecen que el compromiso renal, como primera manifestación de LES, ocupa un segundo lugar en la población joven con relación a los adultos mayores, en los cuales se hace menos frecuente ${ }^{3}$. De igual manera se considera la menor edad un factor de riesgo para padecer de NL severa.

Con relación al sexo y características sociodemográficas, hay estudios retrospectivos en población americana, que establecen que la prevalencia de NL es 4 veces más alta en mujeres en comparación con los hombres, 4 veces mayor entre afroamericanos comparados con blancos ${ }^{4}$ y que los hombres jóvenes americanos de linaje europeo son menos afines a desarrollar NL. ${ }^{1}$

Aproximadamente, la mitad de los pacientes con NL expresan síntomas o hallazgos paraclínicos de afección renal, siendo la proteinuria el más frecuente, seguidos de sedimento urinario activo y elevación de los niveles séricos de creatinina. Aun así, las manifestaciones clínicas subestiman la severidad del compromiso renal en el paciente con LES, por lo tanto, podemos hallar hasta un $15 \%$ de pacientes asintomáticos con NL clases III, IV ${ }^{5}$, lo cual se conoce como NL silente ${ }^{6,7}$.

En un estudio en población asiática ${ }^{8}$, se estableció que los datos clínicos como proteinuria en rango nefrótico eran extrapolables y se presentaron tanto en nefritis proliferativas como no proliferativas. Hubo casos de proteinuria en rangos no nefróticos en casi un $49 \%$ de las NL proliferativas, lo que genera cierta indiferencia entre la correlación clínica y los hallazgos inmunohistopatológicos en la NL, esto hace necesaria la realización de la biopsia renal para definir el tratamiento ideal y el pronóstico de la enfermedad.

El síndrome nefrótico puede presentarse con o sin falla renal y aparece mucho más en las NL membranoproliferativas y en las membranosas clase $\mathrm{V}^{9}$, siendo el síndrome nefrótico más característico de la NL clase IV.

Desde el punto de vista fisiopatológico, en los pacientes con NL clase IV activa, es esperable que se presente hematuria, proteinuria, con el consecuente desarrollo de hipertensión, síndrome nefrótico y deterioro de la función renal, con niveles bajos del complemento y elevación de los niveles séricos de anti DNA. Tomando como base la clasificación de la Sociedad Internacional de Nefrología y de la Sociedad de Patología Renal (ISN/RPS), del $2003^{10}, 480$ casos de NL en Japón fueron categorizados, arrojando como resultado $3 \%$ con clase I, $16 \%$ clase II, $13 \%$ clase III, $11 \%$ clase IV-S, $41 \%$ clase IV-G, $16 \%$ clase V y $1 \%$ clase IVG, siendo la clase IV-G la de mayor incidencia con enfermedad renal en estadio 
final ${ }^{11}$, tal como lo publicó Melvin Schwartz et al., ${ }^{12}$ en su trabajo de reclasificación de acuerdo con los parámetros de la ISN/RPS.

Nuestra investigación se realizó en una cohorte de pacientes con NL en el Caribe colombiano, con el fin de analizar variables clínicas y demográficas; tales como el sexo y la edad, y su correlación con los resultados de la histopatología e inmunomarcación, y conocer su comportamiento en relación con otros grupos poblacionales, buscando establecer directrices ideales de diagnóstico, tratamiento y seguimiento, en pro de mejorar el pronóstico de nuestros pacientes con LES y presunta afección renal.

\section{Materiales y métodos}

Se realizó un estudio retrospectivo, descriptivo durante los años 2012 y 2013. Se incluyeron pacientes adultos mayores de 18 años con diagnóstico de LES y NL, ingresados en la institución y en cuyo estudio de biopsia renal no se encontraba sobreposición de clase.

El diagnóstico de LES, se hizo basado en los criterios diagnósticos del Colegio Americano de Reumatología ${ }^{13}$ y se estableció la presencia de NL, por la existencia de hematuria (más de 5 eritrocitos por campo de alto poder) y proteinuria persistente mayor a $0,5 \mathrm{gr} / \mathrm{dí}$, en el contexto de un paciente con deterioro clínico y evidencia de glomerulonefritis mediada por complejos inmunes compatibles con NL en la biopsia renal ${ }^{14}$.

El estudio se realizó en la Clínica de la Costa, una institución de IV nivel de atención en salud, ubicada en la ciudad de Barranquilla, Colombia. Es un centro de referencia de pacientes con enfermedad renal del Caribe colombiano,

Las biopsias renales fueron interpretadas por el servicio de nefropatología de la institución, de acuerdo con la ISN/RPS $2003^{10,16}$.

La recolección de los datos se realizó directamente de los archivos de dicho servicio y de la historia clínica electrónica de cada paciente a través del Software de manejo de historias clínicas de la institución.
Las variables analizadas fueron sexo, edad, variables clínicas (tensión arterial, hematuria) catalogándose de hipertensión al hallazgo de cifras tensionales mayores de 139/89mmhg, de acuerdo con el séptimo reporte del Comité Nacional Conjunto en prevención, detección, evaluación y tratamiento de la hipertensión arterial. Se definió como hematuria en el sedimento activo, la presencia de 5 o más eritrocitos por campo, de acuerdo con las guías de tratamiento y manejo de la NL del Colegio Americano de Reumatología. Fueron variables paraclínicas la proteinuria de 24 horas, la creatinina sérica, el BUN sérico. Se analizó el perfil inmunológico (ANAS, C3, C4, anti DNA, anti SM, anti-LA, anti-RO), la clase de NL, el índice de actividad y cronicidad e inmunofluorescencia (IgA, IgG, IgM, C3, C4, C1q, cadenas lambda, kappa, albúmina y fibrinógeno) y los patrones de depósito de cada inmunomarcador.

El estudio contó con la aprobación del Comité de Ética de la División de Ciencias de la Salud de la Universidad del Norte.

El análisis estadístico se realizó con el Software estadístico IBM-SPSS (Statistical Package for the Social Sciences) versión 21.

\section{Resultados}

Durante el período comprendido entre enero de 2012 y diciembre de 2014, se diagnosticaron un total de 64 pacientes con NL. En 5 pacientes no fue posible recolectar datos de clasificación histopatológica (2 hombres y 3 mujeres). Seis pacientes correspondían a sobreposición de clase III/V, de los cuales todos eran de sexo femenino.

En total fueron analizados 53 pacientes con diagnóstico definitivo de NL, distribuidos entre las clases II, III y IV (Tabla 1) (figura 1).

La distribución por sexo mostró una mayor frecuencia en las mujeres $(90,5 \%)$ en comparación con los hombres $(9,5 \%)$. La NL clase IV fue la más frecuente $(66,03 \%)$, siendo esta mayor en los hombres $(100 \%)$ que en las mujeres (62,5\%). De acuerdo con la edad, la NL se presentó más entre la tercera y cuarta década de la vida y no se observó un predominio de clase para esta variable (Tabla 1). 


\section{Tabla 1}

Características clínicas y de laboratorio de los pacientes con NL de un centro de referencia del Caribe colombiano durante los años 2012-2013

\begin{tabular}{|l|l|l|l|l|}
\hline \multicolumn{2}{|c|}{ Características } & \multicolumn{3}{c|}{ Clasificación histopatológica* } \\
\cline { 2 - 5 } & II & \multicolumn{2}{c|}{ III } & $32,66(10,46)$ \\
\hline Edad años & $\mathrm{m}(\mathrm{DE})$ & $36,67(14,57)$ & $30,27(6,36)$ & $30(62,5 \%)$ \\
\hline \multirow{2}{*}{ Sexo } & Femenino & $3(6,3 \%)$ & $15(31,3 \%)$ & $5(100 \%)$ \\
\cline { 2 - 5 } & Masculino & 0 & 0 & $19(63,3 \%)$ \\
\hline \multirow{2}{*}{ Hipertensión } & Negativa & $2(6,7 \%)$ & $9(30 \%)$ & $7(70 \%)$ \\
\cline { 2 - 5 } & Positiva & 0 & $3(30 \%)$ & $12(46,2 \%)$ \\
\hline \multirow{2}{*}{ Creatinina } & $<1,3 \mathrm{mg} / \mathrm{dl}$ & $2(7,7 \%)$ & $12(46,2 \%)$ & $18(90 \%)$ \\
\hline \multirow{2}{*}{ Proteinuria } & $\geq 1,3 \mathrm{mg} / \mathrm{dl}$ & $1(5 \%)$ & $1(5 \%)$ & $12(48 \%)$ \\
\cline { 2 - 5 } & $<3,5 \mathrm{gr}$ & $2(8 \%)$ & 0 & $13(100 \%)$ \\
\hline \multirow{2}{*}{ BUN } & $\geq 3,5 \mathrm{gr}$ & 0 & $10(55,6 \%)$ & $7(38,9 \%)$ \\
\hline \multirow{2}{*}{ Hematuria } & $<18 \mathrm{mg} / \mathrm{dl}$ & $1(5,6 \%)$ & $2(10 \%)$ & $17(85 \%)$ \\
\cline { 2 - 5 } & $\geq 18 \mathrm{mg} / \mathrm{dl}$ & $1(5 \%)$ & $1(50 \%)$ & $1(50 \%)$ \\
\cline { 2 - 5 } & Negativo & 0 & $5(16,7 \%)$ & $24(80 \%)$ \\
\hline
\end{tabular}

*No se presentaron pacientes con clasificación histopatológica I, V ni VI

\section{Figura 1}

Prevalencia de la NL en el Caribe colombiano de acuerdo con el género y la clase histopatológica.

\section{Clase NL vs. sexo}

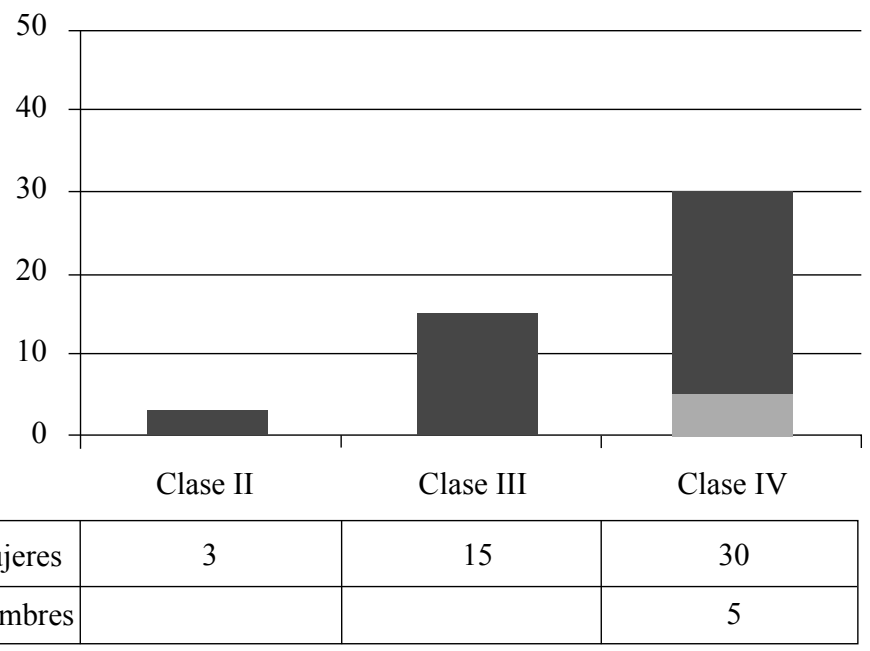


En el grupo de clase IV, la frecuencia de hipertensión arterial fue del $70 \%$, de niveles elevados de creatinina del $90 \%$, de niveles elevados de BUN sérico del $100 \%$, de proteinuria en rango nefrótico del $85 \%$ y de hematuria $80 \%$, que fue mayor que en los grupos II y III (Tabla 1).

De los inmunomarcadores, el más frecuente fue un hallazgo de cadenas lambda (100\%), con una distribución entre las clases II, III y IV del 5,7\%, 28,3\% y $66 \%$, respectivamente; seguido de la $\operatorname{IgM}(94,2 \%)$ con una distribución entre las clases II, III y IV, del $4,1 \%, 30,6 \%$ y $65,3 \%$, respectivamente. La $\operatorname{IgG}$ $(92,4 \%)$ con una distribución entre las clases II, III, IV, del $6,1 \%, 26,5 \%$ y $67,3 \%$, respectivamente.
El fibrinógeno y el C4 marcaron positivos en el $100 \%$ de las veces solo para la NL clase IV, seguidos del C1q (94,1\%) y la albúmina (93,3\%)(Tabla 2).

La hematuria se presentó en un 86,77\% de los casos con marcación positiva para IgA, IgG, IgM, C3, C4, Clq y cadenas lambda, con una desviación estándar de 3,39 a diferencia de los pacientes con marcación positiva para las cadenas kappa en los cuales la creatinina sérica mayor de $1,3 \mathrm{mg} / \mathrm{dl}$, representó el $83,9 \%$ y la hematuria tan solo el $16,1 \%$ del total de casos (Tabla 3).

Finalmente, las variables ANAS, C3, C4, anti DNA, anti SM, anti-LA, anti-RO, que hacían parte del perfil inmunológico, no se tomaron en cuenta para su análisis debido a la falta de datos suficientes para cada una de ellas.

\section{Tabla 2}

Inmunomarcación de los pacientes con NL en un centro de referencia del Caribe colombiano durante los años 2012-2013

\begin{tabular}{|c|c|c|c|c|}
\hline \multirow{2}{*}{\multicolumn{2}{|c|}{ Características }} & \multicolumn{3}{|c|}{ Clasificación histopatológica* } \\
\hline & & \multirow{2}{*}{$\begin{array}{r}\text { II } \\
1(5,3 \%)\end{array}$} & \multirow{2}{*}{$\begin{array}{c}\text { III } \\
4(21,1 \%)\end{array}$} & \multirow{2}{*}{$\frac{\text { IV }}{14(73,7 \%)}$} \\
\hline & Positivo & & & \\
\hline $1 \mathrm{gA}$ & Negativo & $2(6,1 \%)$ & $11(33,3 \%)$ & $20(60,6 \%)$ \\
\hline \multirow{2}{*}{ IgG } & Positivo & $3(6,1 \%)$ & $13(26,5 \%)$ & $33(67,3 \%)$ \\
\hline & Negativo & 0 & $2(50 \%)$ & $2(50 \%)$ \\
\hline \multirow{2}{*}{$\operatorname{IgM}$} & Positivo & $2(4,1 \%)$ & $15(30,6 \%)$ & $32(65,3 \%)$ \\
\hline & Negativo & $1(33,3 \%)$ & 0 & $2(67,7 \%)$ \\
\hline \multirow{2}{*}{$\mathrm{C} 3$} & Positivo & 0 & $13(27,7 \%)$ & $34(72,3 \%)$ \\
\hline & Negativo & $3(50 \%)$ & $2(33,3 \%)$ & $1(16,7 \%)$ \\
\hline \multirow{2}{*}{$\mathrm{C} 4$} & Positivo & 0 & 0 & $14(100 \%)$ \\
\hline & Negativo & $3(7,7 \%)$ & $15(38,5 \%)$ & $21(53,8 \%)$ \\
\hline \multirow{2}{*}{$\mathrm{C} 1 \mathrm{q}$} & Positivo & 0 & $2(5,9 \%)$ & $32(94,1 \%)$ \\
\hline & Negativo & $3(15,8 \%)$ & $13(68,4 \%)$ & $3(15,8 \%)$ \\
\hline \multirow{2}{*}{ Lambda } & Positivo & $3(5,7 \%)$ & $15(28,3 \%)$ & $35(66 \%)$ \\
\hline & Negativo & 0 & 0 & 0 \\
\hline \multirow{2}{*}{ Kappa } & Positivo & $1(2,2 \%)$ & $11(24,4 \%)$ & $33(73,3 \%)$ \\
\hline & Negativo & $2(25 \%)$ & $4(50 \%)$ & $2(25 \%)$ \\
\hline \multirow{2}{*}{ Albúmina } & Positivo & 0 & $1(6,7 \%)$ & $14(93,3 \%)$ \\
\hline & Negativo & $3(8,1 \%)$ & $14(37,8 \%)$ & $20(54,1 \%)$ \\
\hline \multirow{2}{*}{ Fibrinógeno } & Positivo & 0 & 0 & $14(100 \%)$ \\
\hline & Negativo & $3(7,9 \%)$ & $15(39,5 \%)$ & $20(52,6 \%)$ \\
\hline
\end{tabular}




\section{Tabla 3}

Características clínicas y de laboratorio con relación a la inmunomarcación de los pacientes con NL en un centro de referencia del Caribe colombiano durante los años 2012-2013

\begin{tabular}{|c|c|c|c|c|c|c|c|c|c|}
\hline \multirow{2}{*}{\multicolumn{2}{|c|}{ Características }} & \multicolumn{2}{|c|}{ Hipertensión } & \multicolumn{2}{|c|}{ Creatinina (mg/dl) } & \multicolumn{2}{|c|}{ Hematuria } & \multicolumn{2}{|c|}{ Proteinuria (gr/dl) } \\
\hline & & Positiva & Negativa & $<1,3$ & $\geq 1,3$ & Positivo & Negativo & $<3,5$ & $\geq 3,5$ \\
\hline \multirow{2}{*}{ IgA } & Positivo & $3(16,7 \%)$ & $15(83,3 \%)$ & $8(44,4 \%)$ & $10(55,6 \%)$ & $14(93,3 \%)$ & $1(6,7 \%)$ & $9(64,3 \%)$ & $5(35,7 \%)$ \\
\hline & Negativo & $6(23,1 \%)$ & $20(76,9 \%)$ & $23(65,7 \%)$ & $12(34,3 \%)$ & $18(81,8 \%)$ & $4(18,2 \%)$ & $17(58,6 \%)$ & $12(42,44 \%)$ \\
\hline \multirow{2}{*}{$\operatorname{IgG}$} & Positivo & $9(20,9 \%)$ & $34(79,1 \%)$ & $29(58 \%)$ & $21(48 \%)$ & $31(86,1 \%)$ & $5(13,9 \%)$ & $25(58,1 \%)$ & $18(41,9 \%)$ \\
\hline & Negativo & $1(33,3 \%)$ & $2(66,7 \%)$ & $2(50 \%)$ & $2(50 \%)$ & $3(100 \%)$ & 0 & 0 & $2(100 \%)$ \\
\hline \multirow{2}{*}{$\operatorname{IgM}$} & Positivo & $9(21,4 \%)$ & $33(78,6 \%)$ & $30(58,8 \%)$ & $21(41,1 \%)$ & $31(86,1 \%)$ & $5(13,9 \%)$ & $26(61,9 \%)$ & $16(38,1 \%)$ \\
\hline & Negativo & $1(33,3 \%)$ & $2(66,6 \%)$ & $1(33,3 \%)$ & $2(66,7 \%)$ & $2(100 \%)$ & 0 & $1(50 \%)$ & $1(50 \%)$ \\
\hline \multirow{2}{*}{$\mathrm{C} 3$} & Positivo & $10(25 \%)$ & $30(75 \%)$ & $26(55,3 \%)$ & $21(44,7 \%)$ & $31(88,6 \%)$ & $4(11,4 \%)$ & $24(60 \%)$ & $16(40 \%)$ \\
\hline & Negativo & 0 & $5(100 \%)$ & $5(71,4 \%)$ & $2(28,6 \%)$ & $2(66,7 \%)$ & $1(33,3 \%)$ & $3(75 \%)$ & $1(25 \%)$ \\
\hline \multirow{2}{*}{$\mathrm{C} 4$} & Positivo & $5(35,7 \%)$ & $9(64,3 \%)$ & $4(28,6 \%)$ & $10(71,4 \%)$ & $10(83,3 \%)$ & $2(16,7 \%)$ & $7(58,3 \%)$ & $5(41,7 \%)$ \\
\hline & Negativo & $5(16,1 \%)$ & $26(83,9 \%)$ & $27(67,5 \%)$ & $13(32,5 \%)$ & $23(88,5 \%)$ & $3(11,5 \%)$ & $20(62,5 \%)$ & $12(37,5 \%)$ \\
\hline \multirow{2}{*}{$\mathrm{Clq}$} & Positivo & $7(25,9 \%)$ & $20(74,1 \%)$ & $15(45,5 \%)$ & $18(54,5 \%)$ & $24(82,8 \%)$ & $5(17,2 \%)$ & $15(51,7 \%)$ & $14(48,3 \%)$ \\
\hline & Negativo & $3(15,8 \%)$ & $16(84,2 \%)$ & $16(76,2 \%)$ & $5(23,8 \%)$ & $10(100 \%)$ & 0 & $12(75 \%)$ & $4(25 \%)$ \\
\hline \multirow{2}{*}{ Lambda } & Positivo & $10(21,7 \%)$ & $36(78,3 \%)$ & $31(57,4 \%)$ & $23(42,6 \%)$ & $34(87,2 \%)$ & $5(12,8 \%)$ & $27(60 \%)$ & $18(40 \%)$ \\
\hline & Negativo & 0 & 0 & 0 & 0 & 0 & 0 & $12(75 \%)$ & $4(25 \%)$ \\
\hline \multirow{2}{*}{ Kappa } & Positivo & $9(24,3 \%)$ & $28(75,7 \%)$ & $24(54,5 \%)$ & $26(83,9 \%)$ & $5(16,1 \%)$ & $5(17,2 \%)$ & $21(58,3 \%)$ & $15(41,7 \%)$ \\
\hline & Negativo & $1(12,5 \%)$ & $7(87,5 \%)$ & $7(70 \%)$ & $3(30 \%)$ & $7(100 \%)$ & 0 & $6(75 \%)$ & $2(25 \%)$ \\
\hline \multirow{2}{*}{ Albúmina } & Positivo & $4(33,3 \%)$ & $8(66,7 \%)$ & $5(38,5 \%)$ & $8(61,5 \%)$ & $9(81,8 \%)$ & $2(18,2 \%)$ & $5(41,7 \%)$ & $7(58,3 \%)$ \\
\hline & Negativo & $6(18,2 \%)$ & $27(81,8 \%)$ & $26(65 \%)$ & $14(35 \%)$ & $24(88,9 \%)$ & $3(11,1 \%)$ & $22(66,7 \%)$ & $11(33,3 \%)$ \\
\hline \multirow{2}{*}{ Fibrinógeno } & Positivo & $5(35,7 \%)$ & $9(64,3 \%)$ & $4(28,6 \%)$ & $10(71,4 \%)$ & $10(83,3 \%)$ & $2(16,7 \%)$ & $5(41,7 \%)$ & $7(58,3 \%)$ \\
\hline & Negativo & $5(16,1 \%)$ & $26(83,9 \%)$ & $27(69,2 \%)$ & $12(30,8 \%)$ & $23(88,5 \%)$ & $3(11,5 \%)$ & $22(66,7 \%)$ & $11(33,3 \%)$ \\
\hline
\end{tabular}

\section{Discusión}

Esta investigación describe la NL y la correlación clínico e histopatológica en una cohorte de 54 pacientes de la Región Caribe colombiana. Las mujeres tuvieron más NL que los hombres y tanto los adultos jóvenes como los adultos mayores cursaron con la enfermedad, lo cual se diferencia mucho de otros estudios realizados en otras poblaciones, en los cuales no hubo diferencias significativas en cuanto a género ${ }^{1} \mathrm{y}$, de igual manera, de los datos de la literatura mundial que establecen mayor prevalencia de NL en hombres ${ }^{15}$.

De acuerdo con la fisiopatología de la enfermedad ${ }^{12}$, la presencia de hematuria y proteinuria en el estudio de orina, puede ser un marcador de compromiso renal por LES, aunque poco sensible de acuerdo con la evidencia científica ${ }^{5}$, por lo tanto, su ausencia subes- tima, en algunas ocasiones, el grado de compromiso renal por auntoinmunidad.

En esta investigación identificamos a la proteinuria como el hallazgo que se presentó con más frecuencia, mientras que los valores $\geq 3,5 \mathrm{gr} / 24$ horas fueron exclusivos de la clase IV (Gráfico. 2). La presencia o ausencia de hematuria, hipertensión arterial, creatinina sérica $\geq 1,3 \mathrm{mg} / \mathrm{dl}$ y $\mathrm{BUN} \geq 18 \mathrm{mg} / \mathrm{dl}$ no fueron indicadores de una clase de NL específica, de igual manera que una proteinuria $<\mathrm{de} 3,5 \mathrm{gr} / \mathrm{dl}$ no descartó una NL IV. Esto indica que en nuestra población la única manera de establecer el verdadero grado de compromiso renal es través del estudio histopatológico de la biopsia renal y que la ausencia de hematuria, proteinuria, hipertensión arterial, creatinina sérica $\geq 1,3 \mathrm{mg} / \mathrm{dl}$ y $\mathrm{BUN} \geq 18 \mathrm{mg} / \mathrm{dl}$ no descarta clínicamente la presencia de una NL. 


\section{Figura 2}

Relación de las variables clínicas y paraclínicas con las clases de NL en estudio.

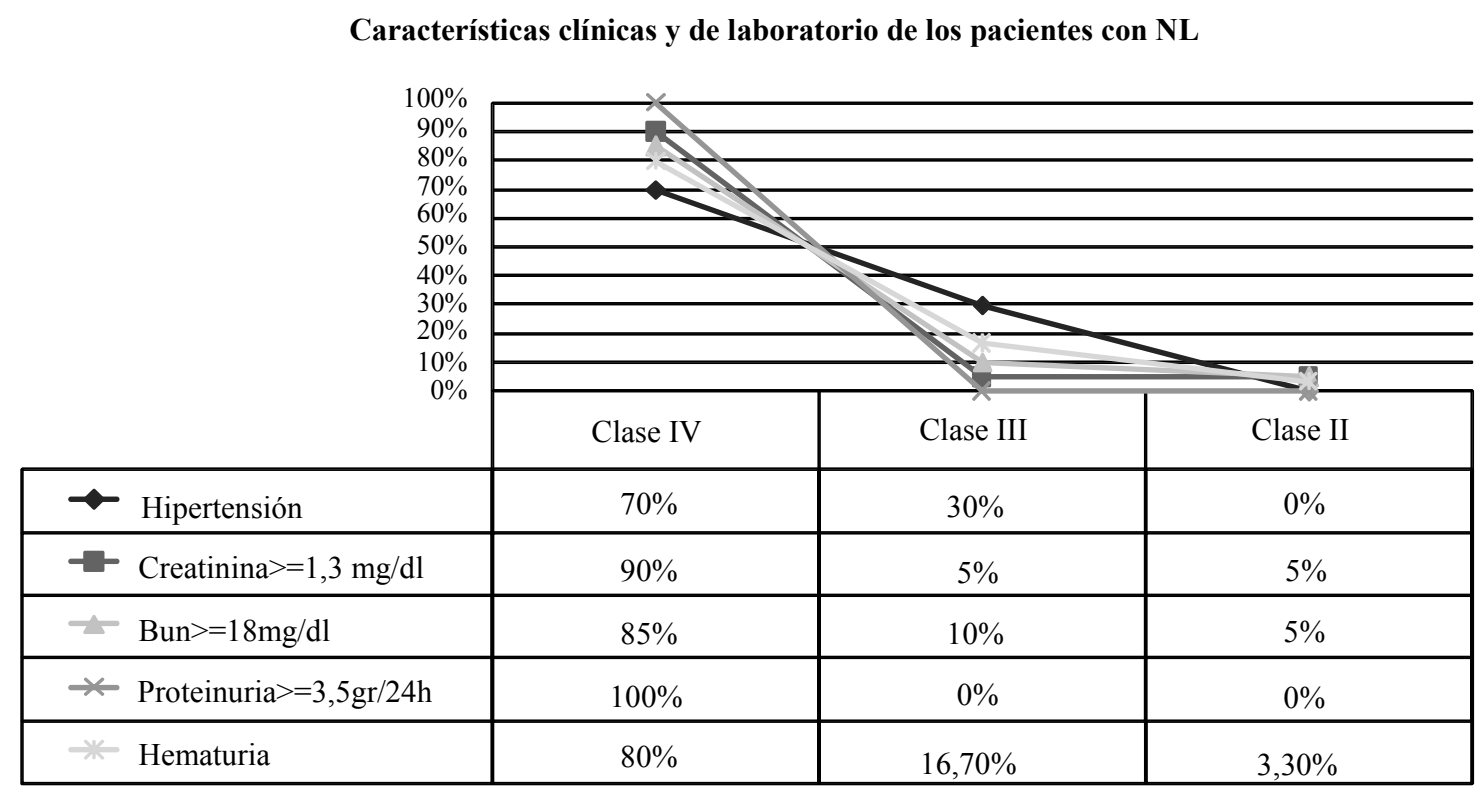

En nuestra cohorte de estudio, el sexo femenino no definió un patrón específico de presentación de la NL y, a pesar que predominó la clase IV en un $62,5 \%$, también hubo distribución entre la II y la III, a diferencia de los hombres en los que en su totalidad hubo presentación de NL clase IV, con lo que podemos argumentar que el sexo masculino no protegería la gran probabilidad de sufrir NL clase IV, sino por el contrario se comportaría como un factor de mayor riesgo.

Hacer este estudio en una institución de referencia para todo el Caribe colombiano, posibilitó contar con una muestra que resultó significativa, pudiendo reflejar el comportamiento de la nefropatía lúpica en esta área del territorio colombiano y dicho conocimiento ser utilizado para generar estrategias de prevención y promoción con relación a este enemigo, el que cada día que pasa, deja de ser más oculto ante la ciencia médica.
Entre las limitaciones del estudio podría mencionarse el tamaño de la muestra, sin embargo, por lo expresado anteriormente se puede considerar, la de nuestra investigación, como representativa de la Región Caribe colombiana. La recolección retrospectiva de la información disponible, puso de manifiesto la ausencia de otros datos, que pudieran ser evaluados conjuntamente con los que forman parte de esta investigación.

\section{Agradecimientos}

A María A Pacheco L, Fabio E Pacheco L, Miguel Holetzeck G, Cristina Saieh D, Guilherme Roca R, estudiantes de Medicina de la Universidad del Norte por su inmensa ayuda en la recolección de datos.

\section{Conflicto de interés:}

Los autores declaran no tener ningún conflicto de interés. 


\section{Bibliografía}

1. Seligman VA, Lum RF, Olson JL, et al. Demographic differences in the development of lupus nephritis: a retrospective analysis. Am J Med 2002;112:726-729.

2. Bastian HM, Roseman JM, McGwin G Jr, et al. Systemic lupus erythematosus in three ethnic groups. XII. Risk factors for lupus nephritis after diagnosis. Lupus 2002;11:152-160.

3. Cervera, Ricard m.d.; Khamashta, Munther a. m.d.; Font, Josep m.d.; Sebastiani, Gian Domenico m.d.; Gil, Antonio m.d.; Lavilla, Paz m.d.; Domenech, Ines m.d.; Aydintug, A. Olcay m.d.; Jedryka-Goral, Anna m.d.; Ramon, Enrique de m.d.; Galeazzi, Mauro m.d.; Haga, Hans-Jacob m.d.; Mathieu, Alessandro m.d.; Houssiau, Frederic m.d.; Ingelmo, Miguel m.d.; Hughes, Graham r.v. m.d., f.r.c.p.; The european working party on systemic lupus erythematosus. Systemic Lupus Erythematosus: Clinical and Immunologic Patterns of Disease Expression in a Cohort of 1,000 Patients. Medicine march 1993;72(2):113-124.

4. Candace H. Feldman, MD, MPH, Linda T. Hiraki, MD, MS, Jun Liu, MD, MPH, Michael A. Fischer, MD, MS, Daniel H. Solomon, MD, MPH, Graciela S. Alarcón, MD, MPH, Wolfgang C. Winkelmayer, MD, ScD, and Karen H. Costenbader, MD, MPH. Epidemiology and Sociodemographics of Systemic Lupus Erythematosus and Lupus Nephritis among U.S. Adults with Medicaid Coverage, 2000-2004. Arthritis Rheum. 2013 March; 65(3):753-763.

5. Wakasugi D, Gono T, Kawaguchi Y, Hara M, Koseki Y, Katsumata Y, Hanaoka M, Yamanaka H. Frequency of class III and IV nephritis in systemic lupus erythematosus without clinical renal involvement: an analysis of predictive measures. J Rheumatol. 2012 Jan;39(1):79-85.

6. Montserrat M. Díaz Encarnación, José Ballarín Castan. Nefropatía lúpica silente. Seminarios de la Fundación Española de Reumatología, Volumen 13, Issue 1, enero-marzo 2012, páginas 3-7.

7. María R. González-Crespo, José I. López-Fernández, Gabriel Usera, María J. Poveda, and Juan J. Gómez-Reino. Outcome of Silent Lupus Nephritis. Seminars in Arthritis and Rheumatism, Vo126, No 1 (August), 1996:pp 468-476.

8. Hsieh YP, Wen YK, Chen ML. The value of early renal biopsy in systemic lupus erythematosus patients presenting with renal involvement. Clin Nephrol. 2012 Jan;77(1):18-24.

9. LM Ortega, DR Schultz, O Lenz, V Pardo and GN Contreras. Lupus nephritis: pathologic features, epidemiology and a guide to therapeutic decisions. Lupus (2010) 19,557-574.

10. Jan J Weening, Vivette D D'Agati, Melvin M Schwartz, Surya V Seshan, Charles E Alpers, Gerald B Appel, James E Balow, Jan A Bruijn, Terence Cook, Franco Ferrario, Agnes B Fogo, Ellen M Ginzler, Lee Hebert, Gary Hill, Prue Hill, J Charles Jennette, Norella C Kong, Philippe Lesavre, Michael Lockshin, Lai-Meng Looi, Hirofumi Makino, Luiz A Moura and Michio Nagata on behalf of the International Society of Nephrology and Renal Pathology Society Working Group on the Classification of Lupus Nephritis. The classification of glomerulonephritis in systemic lupus erythematosus revisited. Kidney International (2004) 65,521-530.

11. Yokoyama H, Okuyama H, Yamaya H. Clinicopathological insights into lupus glomerulonephritis in Japanese and Asians. Clin Exp Nephrol. 2011 Jun;15(3):321-30.

12. Melvin M. Schwartz, Stephen M. Korbet and Edmund J. Lewis (for the Collaborative Study Group). The prognosis and pathogenesis of severe lupus glomerulonephritis. Nephrol Dial Transplant (2008) 23:1298-1306.

13. Michelle Petri, MD, MPH. Review of Classification Criteria for Systemic Lupus Erythematosus. Rheumatic Disease Clinics of North America May 2005. Volume 31. Issue 2, Pages 245-254.

14. Dooley MA, Aranow C, Ginzler EM. Review of ACR renal criteria in systemic lupus erythematosus. Lupus 2004;13:857-60.

15. Bevra H. Hahn, Maureen A. Mcmahon, Alan Wilkinson, W. Dean Wallace, David I. Daikh, John D. Fitzgerald, George A. Karpouzas, Joan T. Merrill, Daniel J. Wallace, Jinoos Yazdany, Rosalind Ramsey-Goldman, Karandeep Singh, Mazdak Khalighi, Soo-In Choi, Maneesh Gogia, Suzanne Kafaja, Mohammad Kamgar, Christine Lau, William J. Martin, Sefali Parikh, Justin Peng, Anjay Rastogi, Weiling Chen, and Jennifer M. Grossman. American College of Rheumatology Guidelines for screening, treatment, and management of lupus nephritis. Arthritis care \& research. vol. 64, no. 6, june 2012, pp 797-808.

16. Markowitz GS, D'Agati VD. Classification of lupus nephritis. Curr Opin Nephrol Hypertens. 2009 May;18(3):220-5. 